\title{
Conduction Mechanism in Electrically Conducting Polymers
}

\author{
Daniel L. Gochnauer \& T. H. Gilani \\ Department of Physics, Millersville University, P. O. Box 1002, Millersville PA-17551 \\ Student:dlg15@,um.edu \\ Mentor:tariq.gilani@millersville.edu
}

\begin{abstract}
The conduction mechanism in conducting polymers is reviewed and experimental results of temperature dependence of electrical conductivity of $\mathrm{PF}_{6}$ doped polypyrrole in temperature range of 77 to $300 \mathrm{~K}$ are discussed. The roomtemperature conductivity was experimentally determined to be $73 \pm 3.4 \mathrm{~S} / \mathrm{m}$ and temperature dependence follows the Mott's variable range hopping model. The average hopping distance at $298 \mathrm{~K}$ was $(6.75 \pm 0.97) \times 10^{-8} \mathrm{~cm}$. The coefficient of decay of the localized states, the density states at the Fermi level, and the hopping activation energy were calculated to be $(3.5 \pm 0.51) \times 10^{7} \mathrm{~cm}^{-1}$, $(1.92 \pm 0.83) \times 10^{22} \mathrm{~cm}^{-3} \mathrm{eV}^{-1}$, and $0.040 \pm 0.001 \mathrm{eV}$ respectively.
\end{abstract}

\section{KEYWORDS:}

Electrically Conducting Polymers; Doped Polypyrrole; Temperature Dependence of Conductivity; Hopping Activation Energy; Density of State at Fermi Level

\section{INTRODUCTION}

The study of electrically conducting polymers, with its many potential applications, deserves recognition as an important frontier in materials science research and development. ${ }^{1-2}$ These conducting polymers have many potential applications. At present they are used as antistatic materials, transparent conductive layers, and protective finishes in printed circuits. Additionally, an increasing focus on organic light emitting diodes and organic polymer solar cells show yet another trend for their future applications. ${ }^{1}$

The electrically conducting polymers have been synthesized with conductivities as high as $10^{3}$ or $10^{4} \mathrm{~S} \cdot \mathrm{cm}^{-1}$, which is merely 1 or 2 orders of magnitude less than that of metals. ${ }^{3-4}$ The conductivity of doped polypyrrole (PPy) can be as high as $10^{4} \mathrm{~S} . \mathrm{cm}^{-1}$. In addition, the higher stability and durability of PPy, makes it an excellent candidate for study. ${ }^{2}$

PPy doped with hexafluorophosphate $\left(\mathrm{PF}_{6}\right)$, hereafter called $\mathrm{PPy}\left(\mathrm{PF}_{6}\right)$, was synthesized and its electrical resistivity was experimentally measured as a function of temperature. The purpose of the study was to develop a better understanding of the conduction mechanism in $\mathrm{PPy}\left(\mathrm{PF}_{6}\right)$.

In metals, the number of valence electrons is minimal compared to the number of available energy orbitals, resulting in higher degrees of freedom allowing the electrons to be delocalized. This enables the electrons to change energy levels within a metal atom, as well as move into the available orbitals in neighboring atoms. In an environment with zero applied electric field, electrons are still moving across the metal surface, but with no net displacement. When an electric field is applied, the electrons still move in random directions, but now there will is a net bias of movement in the direction of the applied electric field. ${ }^{5}$ The same conditions for conductivity are also required in the electrically conducting polymers: delocalized charged carriers and partially filled energy bands. In most organic molecules, however, the valence electrons are localized, due to their participation in the intra-molecular bonds between the atoms. Additionally, due to the covalent nature of nonmetallic bonds, atoms in organic molecules tend not to have the high number of available energy orbitals that metals demonstrate as shown in Figure 1. The solution to this problem lies in the use of conjugated double bonds. A conjugated double bond exists when an organic molecule has alternating single and double bonds, where single and double bonds are defined as chemical bonds between two atoms involving one or two pairs of shared electrons, respectively. The simplest example of a system of conjugated double bonds is trans-polyacetylene, which is also an electrically conductive polymer.

It is the second pair of electrons in the double bonds, which becomes delocalized. One pair of electrons, which holds two atoms together, is denoted as the $\sigma$-bond; any additional pairs are denoted as $\pi$-bonds, which are not required to maintain the bond structure. The $\sigma$-bonds are formed by the two atoms' overlapping s-orbitals and pz-orbitals, parallel to the axis of the bond. The $\pi$-bonds are typically formed by a sharing of electrons between the $\mathrm{p}_{\mathrm{x}}$-and $\mathrm{p}_{\mathrm{y}}$-orbitals, which are perpendicular to the axis of the bond. ${ }^{6}$ For a system with only one double bond, there would be no available orbitals in the adjacent atoms, and so the double bond would be localized. In trans-polyacetylene, however, all of the atoms in the chain have partially filled $\pi$ - bonding $\mathrm{p}$ orbitals available. 




Figure 1. The molecular bondingorbitals for the $1 \mathrm{~s}, 2 \mathrm{p}_{\mathrm{z}}, 2 \mathrm{p}_{\mathrm{x}}$ and $2 \mathrm{p}_{\mathrm{y}}$ orbitals. The $1 \mathrm{~s}$ and $2 \mathrm{p}_{\mathrm{z}}$ orbitals contribute to the single bonding. ${ }^{6}$

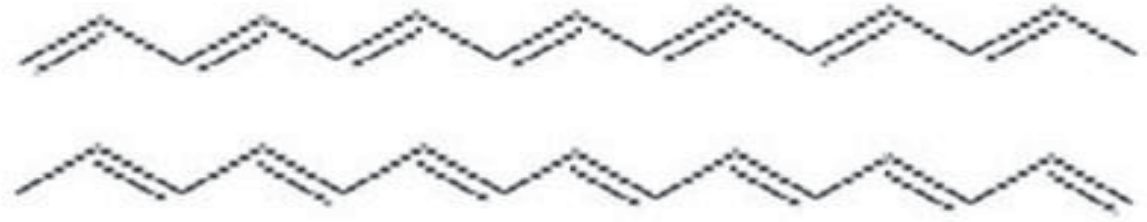

Figure 2. The two forms of trans-polyacetylene are energetically degenerate. In this diagram, each vertex represents a carbon atom, each line represents a bonding pair of electrons, and all of the hydrogen atoms are omitted, as is conventional. ${ }^{7}$

\section{Charge Carriers in Polymers}

Another way to consider this system is as a chain of singly bonded carbons, each with one spare valence electron. Any two adjacent atoms can then share their lone electron to form a double bond; therefore, the energy of such systems as shown in Figure 2 would be lowest. This concept can be extended to the idea that these conjugated, or delocalized, double bonds are able to transport charges effectively within the system. Indeed, such a mechanism for this phenomenon had already been proposed ${ }^{8}$ that charge can be carried by quasi-particles such as solitons, polarons, and bipolarons. A soliton is formed when the chain of double bonds is in a state where there is a single atom, which is not sharing its electron with either of its neighboring atoms. In this state, one of the adjacent atoms can change from its state of $\pi$-bonding with the tertiary atom to a state of $\pi$-bonding with the primary atom in question. These two states are also degenerate, with each other as well as with the states. Thus, this process can occur both naturally and spontaneously. A soliton can become delocalized and is considered to be the most basic charged carrier in conjugated double bond systems. ${ }^{6}$

In other words, it is not likely that a particular electron is moving across the chain of atoms, but rather, it is simply the order of pairing combinations in the polymer, w h i c h changes, allowing the soliton to carry the charge along the chain. This is why the soliton, and related particles, such as the polaron and bipolaron are often referred to as quasi- particles. A soliton is like a fermion in that it has a quantum spin value of $\pm 1 / 2$; however, it differs from the traditional charge carrier, electrons, in that it has zero charge.

A polaron is another type of charged carrier in conjugated double bond polymers with spin $\pm 1 / 2$. Unlike the soliton, however, the polaron has an electric charge of $\pm e$. In many of these types of polymers, it is a common practice to dope the polymer with positively or negatively charged ions in order to make it more electrically conducting. Here, "dopant" is used to mean a chemical additive, which may change the properties of the polymer, as opposed to the more common use of the word in semiconductor physics. When the polymer is doped with negative ions, as is most common, some of the carbon atoms on the chain obtain a positive formal charge. ${ }^{1}$ This alteration to the system allows for an additional mechanism for charge transportation; the traditional electron "hole" may transport charge along the polymer chain. The polaron, then, exists as a coupling of this positive charge with the unpaired electron from a soliton. ${ }^{9}$ Unlike a soliton, however, the polaron has a certain length, given by the distance between the coupled electron and hole. This distance decreases as the concentration of dopant anions increases. Also, as expected, the number of polarons continues to increase as more ions are added. 
a)

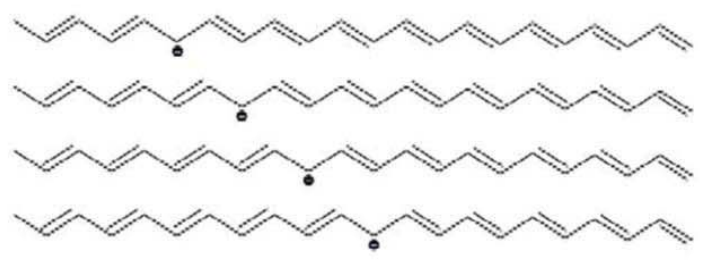

b)

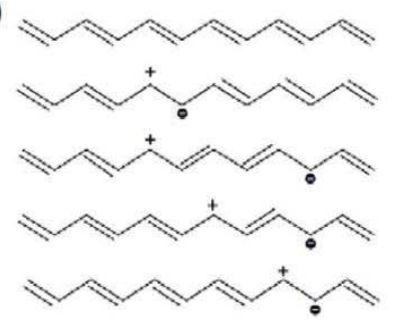

c)

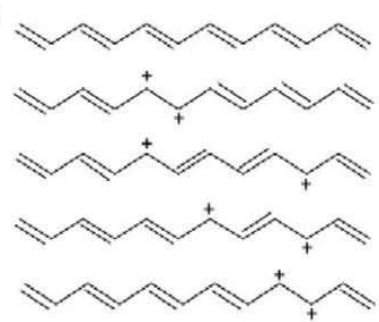

Figure 3. (a) Formation of a soliton in trans-polyacetylene retains the same energetic degeneracy as before. Charge is transported as the soliton hops along the carbon chain.

(b) This diagram shows a mechanism for transportation of a polaron along trans-polyacetylene. The length of the polaron is allowed to vary, as the lone electron and the hole move independently.

(c) The mechanism for bipolaron transportation is analogous to polaron transportation.

Note that in both pictures, however, the counter ions are not shown; they cannot be designated to a specific carbon atom because the positive charge is delocalized. ${ }^{7}$

When the concentration of counter ions increases past a certain point, two polarons may then couple to form another quasiparticle called a bipolaron. ${ }^{8,9}$ Like polarons, bipolarons have a certain length; this length is now defined by the distance between two of the positively charged atoms. Compared to the soliton and the polaron, the bipolaron is unique in that it is a bosonic particle with quantum spin values of 0 or 1 but a bipolaron will have a charge of $\pm 2 e$. The formations of a soliton, a polaron and a bipolaron are depicted in Figure 3.

\section{Band Gap Theory}

The conductive effects of solitons, polarons, and bipolarons can be described effectively using band gap theory. In terms of energy band gap regimes, undoped electrically conducting polymers can be thought of as semiconductors. Therefore, the energy gap between the valence and the conduction band is relatively small, and certain means may be used to allow valence electrons to become excited up into the conduction band. The process of direct ionization requires that an electron must have at least enough energy to jump from the highest occupied energy level to the lowest unoccupied energy level, with no assistance. ${ }^{10}$ In a polaron, however, the lowest unoccupied energy level is increased and the highest occupied energy level decreases, by a value corresponding to the polaron's binding energy. Additionally, the energy gap for a bipolaron is decreased even further, by an amount corresponding to the coupling energy of two polarons. The situations are presented in Figure 4 and Figure 5.

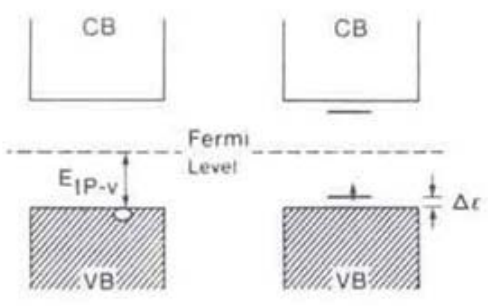

Here, the reference level is the Fermi level, $\mathrm{E}_{\mathrm{IP}-\mathrm{V}}$ is half the energy required for the ionization process, and $\Delta \varepsilon$ corresponds to the binding energy of the polaron. ${ }^{10}$

(a)

(b)

Figure 4. This image compares the energy band gaps for an electrically conductive polymer (a) with no polaron and (b) with a polaron.

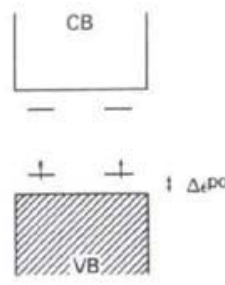

(a)
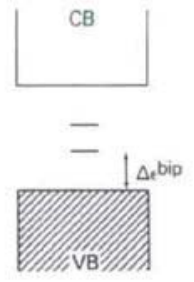

(b)


c o m bined binding energy as well as the coupling energy of the two polarons. ${ }^{10}$

Figure 5. Two polarons would have the same decrease in energy band gap. However, the coupling of two polarons would effectively decrease the gap further. 
This concept can be extended to the idea that polymer conductivity may be dependent on dopant concentration. Dopant concentration is directly related to the number of positively charged atoms along the chain, and therefore the concentration of polarons. Subsequently, with increasing numbers of polarons, there will be more polarons coupling to form bipolarons. For incredibly highly doped polymers, the bipolaron's energy levels spread out into new en ergy bands, in the same way that traditional atomic energy levels form the conduction and valence energy bands. ${ }^{10}$ This results in a minimum energy gap. These energy bands between the original conduction and valence bands allow for a greater population of electrons to carry charge via this new array of available energy levels. This whole process shows how doping electrically conductive polymers can lead to a decreased energy band gap for electronic excitation, which aids in increasing the conductivity of the material.

\section{Variable Range Hopping Model}

Several models ${ }^{11}$ have been proposed to describe the conduction mechanisms in conducting polymers. One of the models that describes quantitatively the transportation of charge within the conducting polymers is Mott's variable range hopping model. ${ }^{12}$ This model assumes that the charge hops along the polymer chain in the form of a soliton as previously discussed, unlike an electron does in a pure metal conductor. Mott's variable range hopping model relates the conductivity $\sigma$ with the temperature, $T$ by the following equation:

$$
\ln \sigma=\ln \sigma_{0}-\left(T_{0} / T\right)^{\frac{1}{4}}
$$

Equation 1.

The characteristic conductivity, $\sigma_{0}$ and the characteristic temperature, $T_{0}$ can be easily obtained from the temperature dependence of the electrical conductivity, $\sigma$. These variables are related to other key parameters by the following additional equations:

$$
\begin{aligned}
\sigma_{0} & =e^{2} R^{2} v_{p h} N\left(E_{F}\right) \\
T_{0} & =\frac{\lambda \alpha^{3}}{k N\left(E_{F}\right)} \\
R & =\left(\frac{9}{8 \pi \alpha k T N\left(E_{F}\right)}\right)^{\frac{1}{4}} \\
W & =\frac{3}{4 \pi R^{3} N\left(E_{F}\right)}
\end{aligned}
$$

Equation 2.

Equation 3.

Equation 4.

Equation 5.

In above equations, $e$ is the charge of an electron $\left(1.602 \times 10^{-19} \mathrm{C}\right), k$ is the Boltzmann constant $\left(8.616 \times 10^{-5} \mathrm{eV} \mathrm{K}-1\right), v_{p h}$ is the phonon frequency, $\lambda$ is a dimensional constant, $a$ is coefficient of decay of the localized states, $R$ is the average hopping distance, $N\left(E_{F}\right)$ is the density of localized states at the Fermi level, and $W$ is the hopping activation energy.

Values of $\sigma_{0}$ and $T_{0}$ can be found by fitting the experimental data of temperature dependence of conductivity to the model given in Equation 1. Then fixing $v_{\mathrm{ph}}$ and $\lambda$, the values of other four unknown variables $\mathrm{R}, N, W$, and $a$ can be obtained algebraically from four equations, Equation 2 through Equation 5. This could be done by hand, but we have tools like Mathematica to do it faster.

\section{EXPERIMENT AND RESULTS}

Incidentally, the process of synthesizing and doping polypyrrole can be carried out in a single reaction. The reaction of pyrrole monomers to form the polymer, polypyrrole is an oxidation reaction, and because that product needs further oxidized for the addition of negatively charged dopants, it is convenient to carry out both reactions in a single electrochemical cell. The reaction is carried out in a an electrochemical cell, consisting of a simple glass beaker, containing a specially prepared solution at $25{ }^{\circ} \mathrm{C}$ for the reaction ${ }^{3,4,13}$ and using a platinum coated anode (approximately $25 \mathrm{~mm}$ x $75 \mathrm{~mm}$ x $1 \mathrm{~mm}$ ). The prepared solution contained

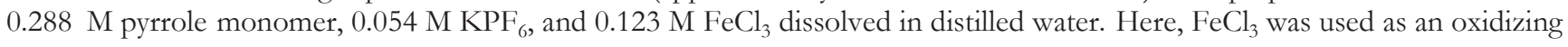
agent. During the synthesis, $4.2 \pm 0.8 \mathrm{~V}$ were applied across the electrodes for 1 to 2 hours, ${ }^{14}$ after which the polymer film is removed from the anode and left to dry at room temperature for another 24 hours. The polymerization process can be represented schematically in Figure 6 (a) and 6 (b).

Various samples were obtained and the best of them were used for the measurements. Typical sample size was $15 \pm 1 \mathrm{~mm} \times$ $3.5 \pm 0.5 \mathrm{~mm} \times 0.010 \pm 0.005 \mathrm{~mm}$. The four-probe method was used to measure resistance at atmospheric pressure while varying the temperature from $77 \mathrm{~K}$ to $300 \mathrm{~K}$. The schematic diagram of the four-probe method is shown in Figure 6 (c). 


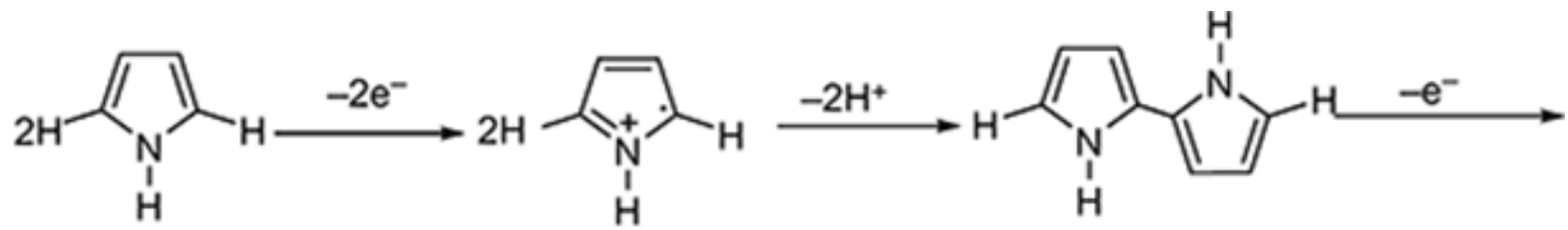

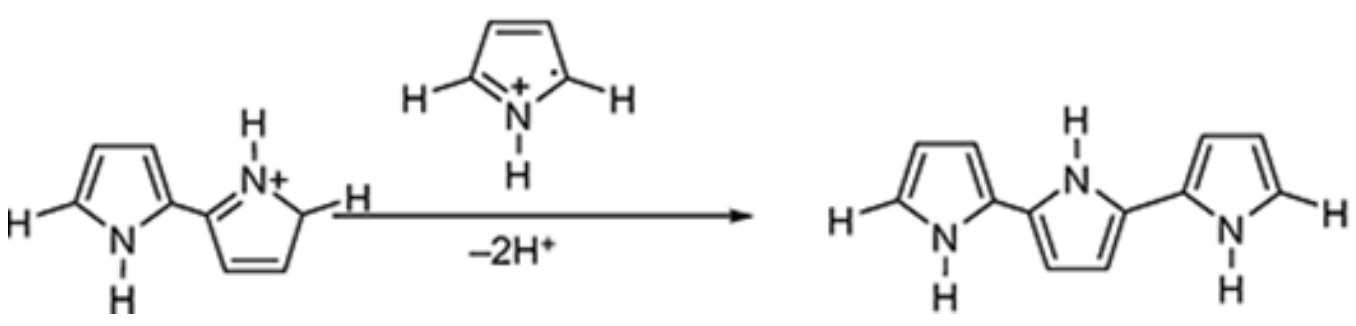

Figure 6 (a) Polymerization of polypyrrole.

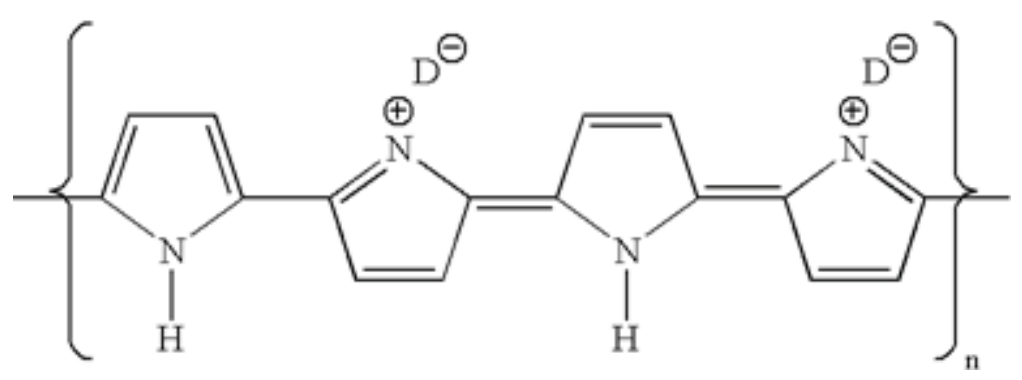

Figure 6 (b) Schematics of doped polypyrrole with an arbitrary dopant ion D.

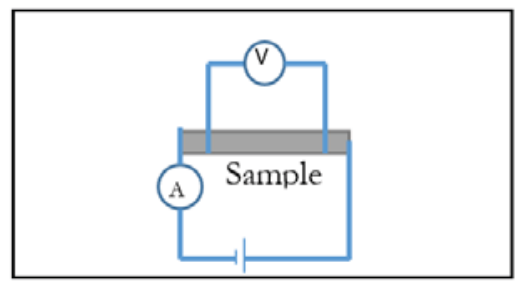

Figure 6 (c) Schematic diagram of the four-probe method for resistance measurement.

The room temperature resistance measurements show Ohmic behavior of the sample at low voltage and current range as shown in Figure 7. The electrical conductivity at room temperature $(298 \mathrm{~K})$ and atmospheric pressure was $73 \pm 3.4 \mathrm{~S} / \mathrm{m}$, obtained from the graph shown in Figure 7.

The temperature dependence of electrical conductivity is shown in Figure 8, where $\ln (\sigma)$ is plotted versus $\mathrm{T}^{-1 / 4}$. A straight line is obtained as suggested by Mott's variable range hopping model. ${ }^{12}$ The corresponding equations (Equation 2 to Equation 5) were used to determine the values of the average hopping distance, $R$, the density of localized states at the Fermi level, $N\left(E_{F}\right)$, and the hopping activation energy, $W$, and the coefficient of decay of the localized states, $a$. Note that the temperature of liquid nitrogen, $77 \mathrm{~K}$, was not actually achieved; this was simply because the sample was not directly in contact with the nitrogen, and so the system could not cool all the way to $77 \mathrm{~K}$. 


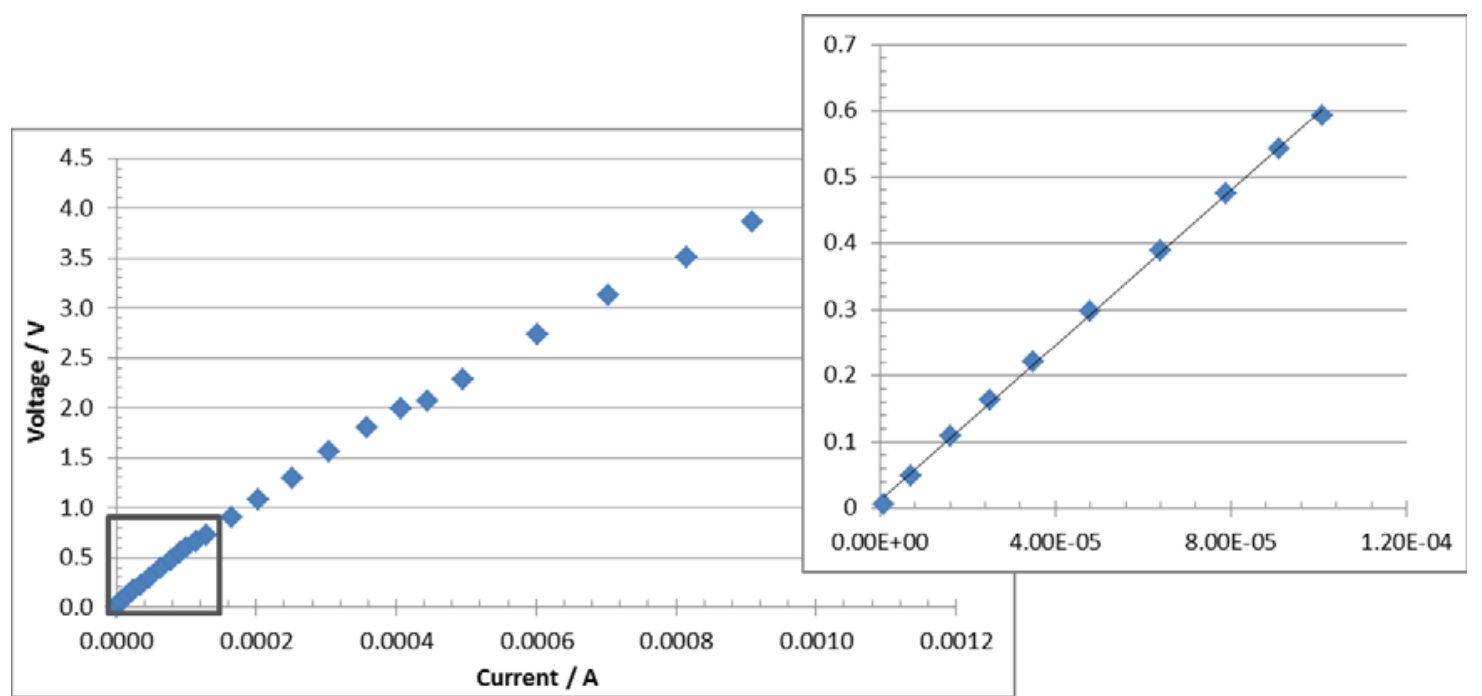

Figure 7. Voltage vs current behavior of $\mathrm{PF}_{\overline{6}}^{-}$doped polypyrrole at room temperature. The region in the square is enlarged and clearly shows Ohmic behavior.

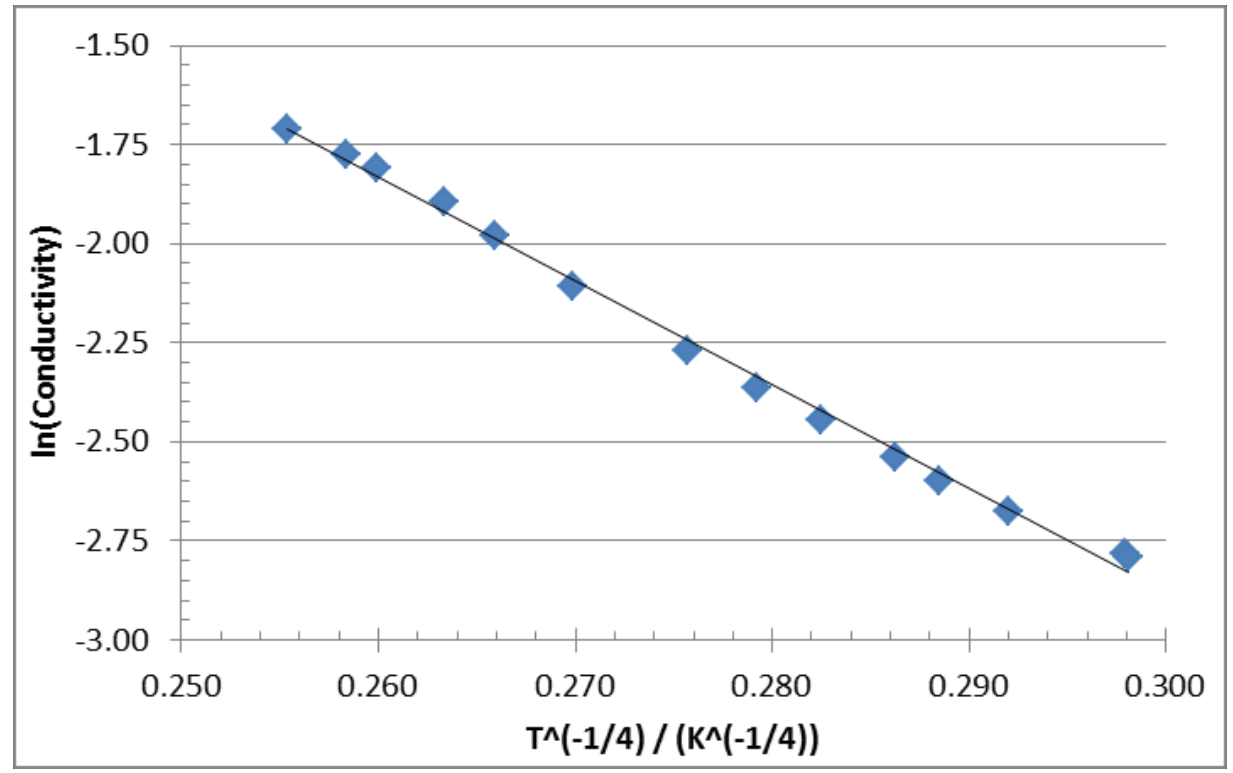

Figure 8. Temperature dependence of conductivity of $\mathrm{PF}_{6}^{-}$doped Polypyrrole, which fits Mott's variable range hopping model given in Equation 1.

\section{DISCUSSIONS}

From Figure 8, along with Equation 1, the characteristic conductivity, $\sigma_{0}$, and the characteristic temperature, $T_{0}$, were determined to be $140 \pm 20 \mathrm{~S} / \mathrm{cm}$ and $(4.7 \pm 0.3) \times 10^{5} \mathrm{~K}$, respectively. The values were subsequently used along with Equation 2 through Equation 5 to determine the values of the average hopping distance, $R$, the density of localized states at the Fermi level, $N\left(E_{F}\right)$, and the hopping activation energy, $W$. The phonon frequency, $v_{p b}$, was set to be $10^{13} \mathrm{~Hz}$ and the dimensional constant, $\lambda$, was set to be 18.1.12

Expressed in terms of the known parameters and the returned fit parameters, the expressions for $\mathrm{R}, N, W$, and $a$ (given in Equation 2 through Equation 5) can also be re-written as following:

$$
\begin{aligned}
R & =\frac{3 \sqrt{3} e^{2} v_{p h} \lambda^{1 / 4}}{4 \times 2^{1 / 4} k \pi^{3 / 4} T^{3 / 4} T_{0}{ }^{1 / 4} \sigma_{0}} \\
N & =\frac{16 \sqrt{2} k^{2} \pi^{3 / 2} T^{3 / 2} \sqrt{T_{0}} \sigma_{0}{ }^{3}}{27 e^{6} v_{p h} \sqrt{\lambda}} \\
\alpha & =\frac{2 k \sqrt{2 \pi} \sqrt{T} \sqrt{T_{0}} \sigma_{0}}{3 e^{2} v_{p h} \sqrt{\lambda}}
\end{aligned}
$$

Equation 6.

Equation 7.

Equation 8. 


$$
W=\frac{k\left(\frac{2}{\pi}\right)^{1 / 4} T^{3 / 4} T_{0}{ }^{1 / 4}}{\sqrt{3} \lambda^{1 / 4}}
$$

Equation 9.

We start with the assumption that the solitons are the primary charged carriers in conducting polymers. The average hopping distance $R$, the density of states at the Fermi level $N\left(E_{F}\right)$, the hopping activation energy $W$, and the coefficient of decay of the localized states $a$ were then calculated and following values were obtained at $298 \mathrm{~K}$ temperature:

$$
\begin{aligned}
& \mathrm{R}=(6.75 \pm 0.97) \times 10^{-8} \mathrm{~cm} \\
& N\left(\mathrm{E}_{\mathrm{F}}\right)=(1.92 \pm 0.83) \times 10^{22} \mathrm{~cm}^{-3} \mathrm{eV}^{-1} \\
& W=0.041 \pm 0.001 \mathrm{eV} \\
& \text { And } \quad a=(3.50 \pm 0.51) \times 10^{7} \mathrm{~cm}^{-1}
\end{aligned}
$$

The mechanism of charge transportation in doped polypyrrole follows the pattern of Mott's variable range hopping model, supported by the experimental data shown in Figure 8. Additionally, the data shows that conductivity increases as temperature increases. This is the behavior of a typical semiconductor, which can be explained by Band gap theory. The values of the calculated parameters all fall within the same order of magnitude when compared with the corresponding values calculated in References 12 and 15. Although these references represent different polymers and dopants, it shows that the results of this experiment fall in the same range. These references view the conduction mechanism in electrically conducting polymers as charge being carried by polarons and the parameters have been calculated based on doped-polyacetylene while the charged carriers in doped PPy are thought to be bipolarons. ${ }^{9}$ However, it is not clear if a bipolaron hops itself or if it breaks into two polarons before hopping. Two polarons can then recombine to form a bipolaron after hoping. Since we obtained parameters, especially average hopping distance, in the same range as calculated for polarons in references 12 and 15, we can conclude based on experimental results that the charged carriers in doped-PPy are polarons with the average hopping distance of approximately $(6.75 \pm 0.97) \times 10^{-8} \mathrm{~cm}$. We must note that the parameter values obtained here are based upon studies on polypyrrole, while polyacetylene is another class of electrically conducting polymers. However, polyacetylene and polypyrrole have very different backbone structure.

\section{ACKNOWLEDGEMENT}

This project was supported by funding from the Millersville University Physics Department as well as the Millersville Student Research Grant Program. Necessary facilities and equipment have also been made available through Millersville University. Additionally, the assistance provided by Mr. Shawn Reinfried has been most helpful. The platinum electrode was generously supplied by Mark Geusic, President of Optimum Anode Technologies.

\section{REFERENCES}

1. Walton, D. (1990) Electrically Conducting Polymers, Materials and Design, 11, 142-152.

2. Skotheim, T. A., Elsenbaumer, R. L., Reynolds, J. R. (1998) Handbook of Conducting Polymers, Eds., 2nd Ed., Marcel Dekker, New York and references there in.

3. Yamaura, M., Hagiwara, T., Iwata, K. (1988) Synth. Met. 26, 209-212.

4. Hagiwara, T., Hirasaki, M., Sato, K., Yamaura, M. (1990) Synth. Met. 36, 241-246.

5. See, for example, Stephen Elliott (2000) The Physics and Chemistry of Solids, Wiley

6. SparkNotes: Molecular Orbital Theory http:/ / wmw.sparknotes.com/ chemistry/ bon ding/molecularorbital/ section1.rbtml Accessed on Feb. 2014

7. Nordén, B. (2000, October 12) Conductive Polymers - a Surprising Discovery http:/ / www.surfacefinishing.com/doc/ conductive-polymers-a-surprising-discovery-0001 Accessed on Feb. 2014

8. Gilani, T. (Dec.16-18, 1997) Transport Properties of dopedPolypyrrole, Frontiers in Physics, Proceeding of $6^{\text {th }}$ National Symposium on Frontiers in Physics, Quaid-i-Azam Univ., Islamabad, Pakistan, 123-130.

9. Gilani, T., Ishiguro, T. (1996) Low-temperature metallic conductance in $\mathrm{PF}_{6}$-doped Polypyrrole, Synth. Metals, 78, $327-331$.

10. Brédas, J. and Street, G. (1985) Polarons, bipolarons, and solitons in conducting polymers, Acc. Chem. Res., 18, $309-315$.

11. Gilani, T. (2005) Evidence for Metal-like Electronic Contribution in Low-temperature Heat Capacity of Polypyrrole, J. Phys. Chem. B, 109, 19204 and reference there in.

12. Kaynak, A. (1998) DC Conduction in Electrochemically Synthesized Polypyrrole Films, Tr. J. Chemistry, 22, 81-85.

13. Chitte, H., Bhat, N., Walunj, V. and Shinde, G. (2011) Synthesis of Polypyrrole using Ferric Chloride $\left(\mathrm{FeCl}_{3}\right)$ as oxidant together with some dopants for use in gas sensors, J. Sensor Technology, 1, 47-56.

14. Shoa, T., Cole, M., Munce, N., Yang, V. and Madden, J. (2007) Polypyrrole operating voltage limits in aqueous sodium hexafluorophosphate, Proc. SPIE, 6524, 6524211-6524218.

15. Vernitskaya, T. and Efimov, O. (1997) Polypyrrole: a conducting polymer; its synthesis, properties and applications, Rus. Chem. Rev., 66, 443-457. 


\section{ABOUT THE STUDENT AUTHOR}

Daniel Gochnauer graduated from Millersville University, PA in 2014 with B. Sc. in Physics and Chemistry, and is now a graduate student at the University of Washington.

\section{PRESS SSUMMARY}

The development of electrically conducting polymers maintains its worth as a valued field of materials science and solid state physics. The focus in this article is conduction mechanism in electrically conducting polymers. A short-review of the conduction mechanism is presented. Also experimental results of temperature dependence of electrical conductivity of $\mathrm{PF}_{6}$ doped polypyrrole in temperature range of 77 to $300 \mathrm{~K}$ are discussed in reference with Mott's variable range hopping model. The temperature dependence of conductivity follows the Mott's model. The average hopping distance, the density states at Fermi level and the hopping activation energy were determined from the experimental data. 